\title{
AN ANALYSIS OF REMOTE SENSING SENSOR ISSUES AND SENSOR CROSS CALIBRATION
}

\author{
Sheron Henry Christy \\ Department of Electronics \& Communication \\ V.V.P. Engineering College, Rajkot, Gujarat, India.
}

\begin{abstract}
Remote sensing is a very good alternative technology for managing natural resources as compared to conventional technologies. This paper highlights the various challenges in UAS sensors. Comparison of IRS-P6 and Land Sat sensors is described from accuracy point of view by covering same areas by both the sensors which gives the performance features of both the sensors. Inter sensor calibration is depicted to realize its importance in applications like precision farming, disaster management, etc. requiring multiple dated satellite images.
\end{abstract}

Keywords-Remote Sensing, UASs, cross calibration, sensors, artifacts

\section{INTRODUCTION}

Remote sensing [1] is a technology concerned with measurement of physical properties using reflected/emitted energy of distant objects. It is a method using electromagnetic wave for identifying features of earth surface and estimation of their geo-biophysical properties. Target classification is assisted by major features like spectral, spatial, temporal and polarization impressions of the target/sensor. Earth surface data as observed by the sensors in different reflected, scattered and/or emitted wavelengths is geometrically and radio metrically rectified before spectral detail extraction. Remote Sensing data helps managing natural resources better than conventional technologies due to its capability for a repetitive coverage, compact view and with calibrated sensors to survey at different resolutions and detect changes. Therefore, it is quite useful to study various characteristics, challenges and performances of the sensors. In [2], identification of advantages of various UAS platforms and sensor capabilities as pertinent to the demands of stakeholders in the scientific research zone has been discussed. In [3], discussion has been done on apex of quantitative perception using a selected UAS for collecting powerful data on composite forest environments. In [4], Inter calibration methods providing an experimental means of identifying and correcting comparative biases in radiometric calibration between satellite instruments are discussed. In [5], vicarious calibration making utilization of Earth's "invariant" natural targets for the post-launch calibration of sensors is discussed.

\section{UAS SENSOR CHALLENGES}

\section{A. Camera Shortcomings}

There are various issues with UASs [6] that require to be addressed for their full potential to be realized for environmental monitoring and measurements. Geometric and radiometric constraints imposed by current generation of lightweight and consumer-grade digital cameras are chief among them. These are designed for the overall market and are not optimized for remote sensing and photogrammetric applications. Higher-end equipments tend to be too bulky to be used with current lightweight UASs. There's still calibration consistency problem with conventional sensors

Spectral constraints cover spectral response curves from consumer-grade cameras which are normally poorly calibrated, making it hard to transform brightness values to radiance which is vital for comparative studies. Another drawback is that a lot of consumer cameras are susceptible to vignetting, where the sides of images appear darker than the centres. This effect occurs because rays of sunshine at the sides of the image need to undergo a greater optical thickness of the optical lens. Thus, they are strongly attenuated in image's centre than light rays. Although there is a possibility to cover up many of those effects and to make a mosaic that appears seamless, unplanned color balancing may impact the performance of automated image classification computations. These constraints degrade the standard of the spectral details which can be recovered from a typical UAS survey. Consumer-grade cameras' geometry also presents challenges in which it is often difficult to get reliable calibrations for such cameras even under ideal conditions. Alternatively, one can use a micro-four-thirds format camera. This class of cameras has similar features to a full single lens camera, but with a much smaller body.

\section{$B$. Image Classification}

UASs can be utilized to gather aerial images at a considerably higher spatial resolution even at centimeter level. While this resolution gives offering variety of 
benefits, the quantity of detail presents new challenges from the purpose of view of image classification. The brightness of an individual pixel represents an aggregate of the reflected radiation from the various cover types making up that pixel at resolutions of only few centimeters. With separate pixels which depict branches, leaves and underlying ground cover, the individual component sections of plants and trees frequently become apparent. Due to high contrast differences between these features, mixed pixels, comprising various combinations of those components, will also tend to point out greater variation than would be apparent for lower resolution imagery. In such circumstances, pixel-based image classification algorithms may not give good results.

\section{Illumination Issues}

Variations between shaded and sunlit areas are often remarkable on a fair sunny day, particularly where there are cumulus clouds overhead giving sharp welldefined shadows. Such circumstances can pose significant challenges for the automated image matching computations utilized triangulation and digital elevation model generation from experience. Another illumination effect is that the presence of image hotspots, where a bright spot appears within the image. These are due to the consequences of bidirectional reflectance, which depends on the relative position of the image sensor and therefore the sun.

\section{Relief Displacement}

Because of the low flying heights used, UAS imagery is especially susceptible to the consequences of relief displacement. For non vegetated areas, such displacement is seperated during the ortho rectification process, assuming that the DSM/DTM used accurately represents the terrain. It becomes more complicated when handling trees and buildings. In these cases, local displacement is usually considerable, and there can often be hidden areas where no data has been captured. If a DSM is employed to ortho rectify such images, the result can often be a choppy looking, irregular image. Due to the noise present within the surface, employing a DTM will typically result in a smoother looking image; however, locally elevated features will often still be subject to the consequences of relief displacement. As such it's often difficult to supply a real ortho image, which accurately represents all features.

\section{E. Mosaic Artifacts}

The production of mosaics can cause additional problems. Image artifacts are often created where the color-balancing algorithms fail to figure properly. These can arise where the contrasts of individual image bands fall outside the span of the image histograms used for image matching. Another common occurrence is striping on the ultimate mosaic. this may often occur where there's insufficient overlap between flight lines to permit color matching to be administered successfully.

\section{IRS-P6 AND LANDSAT SENSORS}

The image sets from LISS-III and P6 AWiFS sensors [7] were correlated to images from L7 ETM+ and L5 TM sensors for perception of their radiometric calibration precision. In this technique, calibration of surface observations assisted image statistics from areas observed nearly concurrently by the 2 sensors was performed. The distinctive reflectance estimates acquired from these sensors acknowledged within 13\%. Artifacts were recognized within the areas of overlap, both of the P6 AWiFS quadrants and of the scan-gap L7 ETM+mosaic. The precursory results specified that the IRS-P6 sensors can be cross calibrated to the Landsat sensors within a precision of $13 \%$. Reflectance of analogous regions viewed by the L5 TM plotted against equivalent regions viewed by P6 LISS-III were within 5.5\% covering all bands excluding band 2 , which had a $16.4 \%$ difference. The cross calibration of AWiFS to the L5 TM induced the simplest outcome, with similar differences in absolute calibration of roughly $6 \%$ covering all bands of the TM and AWiFS sensor as the AWiFS image was present in both collections and the calibration of the AWiFS sensor is usually nearer to the Landsat sensors than to LISS-III. The anticipated difference in outcomes from those applications covering the NLCD product generation was found to similar to cross calibration accuracies. Often this is a precursory measure of the feasibility of using non-Landsat sensors for Landsat data continuity in scientific applications. For some classes L5 was typically 5\% or more precise than LISS-III and P6 AWiFS. These happen on the classification of evergreen, shrub/scrub, emergent wetlands and woody wetlands. Classically, L5 TM bands 1 and 7 have been extensively utilized for vegetation and moisture differentiation which has increased toughness with LISS-IIII and P6 AWiFS to classify moist and dry vegetation. Individual LISS-III and P6 AWiFS products were a bit less precise in categorizing particular land-cover classes but gave a very beneficial estimation to L7 ETM+ and L5 TM for percent impermeable surface and percent tree cover.

\section{SENSOR CROSS CALIBRATION}

For applications like precision farming, disaster management, etc, it's required to watch events using multidate satellite imagery acquired over an equivalent area. This need is often best met with a set of satellites whose radiometric features are completely modeled. In spite of 
having series of satellites with similar or analogous radiometric features, systematic difference between their measured radiance existed because of intrinsic variation in observing sensor response and geometries. Inter-sensor calibration [8] exercises are administered to determine radiometric relationship between the sensors of comparable spectral bands in order that their products are often utilized in conjunction with the other for deriving the change in events. Based on assumption that the atmospheric and surface properties were relatively consistent between the 2 data acquisitions and using prelaunch coefficients, the target's top-of atmosphere radiance was calculated from these data sets. These data sets' spectral response curves for each sensor displayed a resultant inherent difference which was standardized bandwise typically with the pre-launch measured data. Multiple data sets acquired at different dates were utilized to evaluate this coefficient to form it usable for all the seasons. For authenticating precision of the inter-sensor coefficients acquired from this technique, a new dataset was taken. After utilizing inter-sensor calibration methodology, an honest association was found to exist among the data sets of the sensors. Approximately flat regions within the desert were chosen with a selection basis of having standard deviation of the window less than 3 counts for the investigation and taking average of several windows which were absolutely nearby geometrically. The scaling factor $\mathrm{m}$ was provided to convert IRS-P6 radiance to correspondent of IRS-1D, and from IRS-1C to IRS-1D calculated from the near synchronous and synchronous or pass scenes. Additionally, derived multiplier from the IRS-P6 to IRS-1C was provided. Before and after applying the cross-calibration results, difference in radiances was computed between IRS-P6 and IRS-1D. After calibration, the slope was inclining toward 1.0 while the offset value was minimized in observation. Thus, wide field sensors of the P6, IRS-1D and 1C spacecrafts with near-synchronous and synchronous matching scenes were tried to get crosscalibrated by this exercise. Intrinsic inter-sensor spectral response variations were reimbursed and recognition of uniform flat response regions over the Thar desert scene was observed. The one-to-one correspondence in spectral radiance for the data set selected in observations was enhanced by this effort.

\section{CONCLUSION}

UAS sensors find many challenges in camera shortcomings, illumination issues, image classification, relief displacement and mosaic artifacts to explore their full potential in remote sensing. While comparing IRS-P6 sensors with Landsat sensors, slightly less accuracy is observed in IRS-P6 sensors covering same areas which can be addressed by more image acquisition and potential 5 day revisits by IRS-
P6 sensors. Inter sensor cross calibration may prove to be helpful in important applications like disaster management and precision farming.

\section{REFERENCE}

[1] P. S. Roy, M. D. Behera and S. K. Srivastav, "Satellite Remote Sensing: Sensors, Applications and Techniques", Proceedings of The National Academy of Sciences, Sect. A Phys. Science, vol 87, issue 4, pp. 465-472, November 2017.

[2] Adam C. Watts, Vincent G. Ambrosia and Everett A. Hinkley, "Unmanned Aircraft Systems in Remote Sensing and Scientific Research: Classification and Considerations of Use", Remote Sensing Journal, MDPI, pp. 1671-1692, 2012.

[3] Benjamin T. Fraser and Russell G. Congalton, "Issues in Unmanned Aerial Systems (UAS) Data Collection of Complex Forest Environments", Remote Sensing Journal, MDPI, pp. 1-21, June 2018

[4] Gyanesh Chander, , Tim J. Hewison, , Nigel Fox, Xiangqian Wu, Xiaoxiong Xiong, , and William J. Blackwell, "Overview of Intercalibration of Satellite Instruments", IEEE Transactions on Geoscience And Remote Sensing, vol. 51, issue. 3, pp 1056-1080, March 2013

[5] Richard Müller, "Calibration and Verification of Remote Sensing Instruments and Observations", Remote Sensing Journal, MDPI, pp 5692-5695, 2014.

[6] Ken Whitehead and Chris H. Hugenholtz, "Remote sensing of the environment with small unmanned aircraft systems (UASs), part 1: a review of progress and challenges", Journal of unmanned vehicle system, pp 69-85, July 2014.

[7] Gyanesh Chander,, Michael J. Coan, and Pasquale L. Scaramuzza, "Evaluation and Comparison of the IRS-P6 and the Landsat Sensors", IEEE Transactions On Geoscience And Remote Sensing, Vol. 46, Issue 1, January 2008.

[8] Srinivas, S. Jayabharathi, S. Muralikrishnan And A. Senthil Kumar, "Cross-Calibration Of IRS-1C, -1D And -P6: Wide Angle Sensors Using Synchronous Or Near Synchronous Matching Scenes", Symposium Of ISPRS Commission IV, Goa, India, Vol XXXVI, Part 4, September 2006 